\title{
Spectral $\mathrm{H}_{2}$ optimal correction of additive fault estimation observer
}

\author{
Evgeny I. Veremey ${ }^{1}$, Yaroslav V. Knyazkin ${ }^{1, \text { a }}$ \\ ${ }^{1}$ Saint-Petersburg state University, Faculty of Applied Mathematics and Control Processes, 199034 Saint-Petersburg, Russia
}

\begin{abstract}
This study is devoted to design of the fault estimation adaptive observer. Initial observer is modified with correction filter in order to improve robustness against external disturbance with known spectral structure. Computation of correctors parameters is based on special spectral algorithm of SISO (Single Input and Single Output) $\mathrm{H}_{2}$ optimisation in frequency domain. Novel algorithm is proposed for practical implementation. Its effectiveness is demonstrated with example of successive additive fault estimation during marine ship yaw motion process.
\end{abstract}

\section{Introduction}

Due to increasing reliability demands of dynamic systems, problem of fault estimation is paid serious attention nowadays. Fast and accurate fault estimation [1$3]$ is necessary for effective fault tolerant control, such as described e.g. in [4]. Fault detection and fault estimation for linear time invariant (LTI) systems is well-studied area. On the other hand, most of existing methods ignore structure of external disturbance. Proposed approach can be treated as the method of observer's correction to avoid false alarms.

Most of the papers, devoted to the fault detection and fault estimation problems, use various approaches based on linear matrix inequalities. It is well known universal technique of numerical solution for control problems. On the other hand, there are many situations when the problem can be solved significantly easier with the help of the frequency domain specific approach, based on polynomial computations.

An application of this approach is demonstrated in this paper, which is organised as follows. In Section 2, structure of initial observer to be modified is presented. In Sections 3, 4 we describe spectral approach to observer correction and its practical realisation. Finally, Section 5 contains conclusion of the paper, overall results and directions for future research.

\section{Problem statement}

Let us consider the LTI system

$$
\begin{aligned}
& \dot{x}=\mathbf{A} x+\mathbf{b} u+\mathbf{E} f+\mathbf{g} d, \\
& y=\mathbf{C} x,
\end{aligned}
$$

where $x \in \mathbf{R}^{n}$ is the system state vector; $y, u, f, d$ are the scalar values : $y$ is the output signal, $u$ is the control, $f$ represents additive fault disturbance and $d$ is the main external disturbance. Suppose that faults are constant or slow varying, i.e. $\dot{f}(t) \approx 0$.

Adaptive fault estimation observer can be described by the following system

$$
\begin{aligned}
\dot{\hat{x}} & =\mathbf{A} \hat{x}+\mathbf{b} u+\mathbf{E} \hat{f}+\mathbf{L}(y-\mathbf{C} z), \\
\dot{\hat{f}} & =\mathbf{l}_{\mathbf{f}}(y-\mathbf{C} z),
\end{aligned}
$$

where $\hat{x} \in R^{n}$ is the estimated state vector, $\hat{f}$ is the estimated scalar fault, $\mathbf{L} \in R^{n}$ and scalar $\mathbf{l}_{\mathrm{f}}$ are observer parameters to be designed. Some solutions of this problems described, e.g. in the papers [1-3].

Let us suppose that the adaptive observer (2) has already been designed for the system with no disturbance. The matter of the discussion is to propose the algorithm of its improvement with the help of corrective filter, compensating the action of regular disturbance with initially given frequency $\omega_{0}$.

Generally speaking, the mentioned base observer may have more complicated structure and $\mathbf{L}, l_{f}$ can not be constant, but transfer functions that do not affect to the main idea. Correction of observer is given by

$$
\begin{aligned}
& \dot{\hat{x}}=\mathbf{A} \hat{x}+\mathbf{b} u+\mathbf{E} \hat{f}+\mathbf{L} v, \\
& \dot{\hat{f}}=\mathbf{I}_{\mathbf{f}} v, \\
& v(s)=W(s)(y-\mathbf{C} \hat{x}),
\end{aligned}
$$

where $W(s)$ is a transfer function of a dynamic corrector to be computed. Let denote $e_{x}=x-\hat{x}, e_{y}=y-\mathbf{C} \hat{x}$,

$$
\begin{array}{lll}
e_{f}=f-\hat{f}, & e=\left(\begin{array}{ll}
e_{x} & e_{f}
\end{array}\right)^{\mathrm{T}}, & \overline{\mathbf{C}}=\left(\begin{array}{ll}
\mathbf{C} & 0
\end{array}\right), \\
\overline{\mathbf{L}}=-\left(\begin{array}{ll}
\mathbf{L}^{\mathrm{T}} & \mathbf{l}_{\mathbf{f}}
\end{array}\right)^{\mathrm{T}}, & \overline{\mathbf{g}}=\left(\begin{array}{ll}
\mathbf{g} & 0
\end{array}\right)^{\mathrm{T}}, \quad \overline{\mathbf{A}}=\left(\begin{array}{cc}
\mathbf{A} & \mathbf{E} \\
0 & 0
\end{array}\right) \quad \text { and let }
\end{array}
$$

${ }^{a}$ Corresponding author : yaroslavknyazkin@gmail.com 
describe an error dynamics of the corrected observer (3) with the following equations:

$$
\begin{aligned}
& \dot{e}=\overline{\mathbf{A}} e+\overline{\mathbf{L}} v+\overline{\mathbf{g}} d, \\
& e_{y}=\overline{\mathbf{C}} e, \\
& v(s)=W(s) e_{y}(s) .
\end{aligned}
$$

It is necessary to compute a transfer function $W(s)$ of dynamic corrector (filter), providing fast and accurate fault estimation in presence of the external disturbance.

\section{Spectral approach to $\mathrm{H}_{2}$ optimisation}

One of the ways to construct disturbance compensative filter is to solve mean-squire optimization problem with the functional

$$
\widetilde{J}=\widetilde{J}(W)=\lim _{T \rightarrow \infty} \int_{0}^{T}\left(e_{y}^{2}+k^{2} v^{2}\right) d t .
$$

The second summand in (5) can be considered as the norm of "velocity" for estimated fault signal due to the fact that $v$ is proportional to its derivative.

Let us convert the system (4) to the form

$$
\begin{aligned}
& A(s) e_{y}(s)=B(s) v(s)+P(s) d(s), \\
& v(s)=W(s) e_{y}(s),
\end{aligned}
$$

where $\quad A(s)=\operatorname{det}(\mathbf{I} s-\overline{\mathbf{A}}), \quad B(s)=A(s) \overline{\mathbf{C}}(\mathbf{I} s-\overline{\mathbf{A}})^{-1} \overline{\mathbf{L}}$, $P(s)=A(s) \overline{\mathbf{C}}(\mathbf{I} s-\overline{\mathbf{A}})^{-1} \overline{\mathbf{g}}$. Let suppose that the external disturbance $d(t)$ has the following spectral power density:

$$
\begin{gathered}
S_{d}(s) \equiv H(s) H(-s), \\
H(s)=\frac{N_{d}(s)}{T_{d}(s)}=\sqrt{\frac{4 D_{r} \alpha}{\pi}} \frac{1}{s^{2}+\alpha s+\alpha^{s}+\beta^{2}},
\end{gathered}
$$

where $D_{r}$ is the dispersion, $\beta$ is the main frequency of the disturbance, $\alpha=s_{t} \beta, s_{t}$ is the blurriness of the spectrum.

The $\mathrm{H}_{2}$ problem solution, described in the paper [5], can be used for computing of the optimal $W(s)$. There are two main reasons to choose this approach. Firstly, it is useful, when spectral structure of external disturbance is known. Moreover, this $\mathrm{H}_{2}$ approach is based on polynomial calculations instead of "2-Riccati" or LMI technique. This can be essentially effective, when computational resources are limited, e.g. for onboard control systems of autonomous mobile robots.

Let us consider the steps of the spectral algorithm of synthesis based on the mentioned approach:

1. Execute the factorization of the polynomials:

$$
\begin{aligned}
& k^{2} A(s) A(-s)+B(s) B(-s) \equiv G(s) G(s), \\
& P(s) P(-s) N_{d}(s) N_{d}(-s) \equiv N(s) N(-s),
\end{aligned}
$$

where $N(s)$ and $G(s)$ are Hurwitz polynomials.

2. Construct the auxiliary polynomial

$$
R(s)=\sum_{i=1}^{n} \frac{G(-s)}{g_{i}-s} \frac{B\left(-g_{i}\right) N\left(-g_{i}\right)}{A\left(g_{i}\right) T_{d}\left(g_{i}\right) G^{\prime}\left(-g_{i}\right)},
$$

where $g_{i}, i=\overline{1, n}$ are the distinct roots of $G(-s)$.

3. Represent a transfer function of the optimal filter

$$
\begin{gathered}
W(s)=W_{0}(s)=\frac{W_{1}^{0}(s)}{W_{2}^{0}(s)}= \\
=\frac{\left[A(s) T_{d}(s) R(s)+B(-s) N(-s)\right] / G(-s)}{\left[A(s) T_{d}(s) R(s)-k^{2} A(-s) N(s)\right] / G(-s)},
\end{gathered}
$$

where division to polynomial $G(-s)$ is done totally.

Consider transfer function $F(s)$ from external disturbance $d(s)$ to $e_{f}(s)$ in closed-loop system with the obtained $W(s)$. The value $\left|F\left(j \omega_{0}\right)\right|$ demonstrates the effectiveness of the filter. If this value is large, i.e. sensitivity of fault estimation to disturbance is too high, it is necessary to compute another filter.

Note that the function $W(s)$ must be a proper fraction to avoid numerical difficulties of implementation. Let us derive expression of degrees of the polynomials $W_{1}^{0}(s)$ and $W_{2}^{0}(s)$ from degrees of polynomials, mentioned in formulas (6),(8)-(10): $\quad n=\operatorname{deg} A(s), \quad m=\operatorname{deg} B(s)$, $p=\operatorname{deg} N(s), \quad q=\operatorname{deg} T_{d}(s)$. Degrees of auxiliary polynomials $R(s), \quad G(s)$ are $\operatorname{deg} R(s)=n-1$, $\operatorname{deg} G(-s)=n-1$, that implies

$$
\begin{aligned}
& \mu_{1}=\operatorname{deg} W_{1}^{0}(s)=\max (n+q+n-1, p+m)-n, \\
& \mu_{2}=\operatorname{deg} W_{2}^{0}(s)=\max (m+q+n-1, p+n)-n .
\end{aligned}
$$

There are three possible cases,

1. if $p \leq m+q-1$, then

$$
\begin{aligned}
& \mu_{1}=n+q-1, \\
& \mu_{2}=m+q-1 ;
\end{aligned}
$$

2. if $m+q-1 \leq p \leq 2 n-m+q-1$, then

$$
\begin{aligned}
& \mu_{1}=n+q-1, \\
& \mu_{2}=p
\end{aligned}
$$

3. if $p \geq 2 n-m+q-1$, then

$$
\begin{aligned}
& \mu_{1}=p+m-n, \\
& \mu_{2}=p .
\end{aligned}
$$

It is obvious, that order $k_{d}$ of numerical derivative of output signal $e_{y}(t)$ can be expressed 


$$
k_{d}=\left\{\begin{array}{l}
0, \text { if } \mu_{1} \leq \mu_{2}, \\
\mu_{1}-\mu_{2}, \text { if } \mu_{1}>\mu_{2} .
\end{array}\right.
$$

Let us calculate $k_{d}$ for every previously mentioned case (12)-(14). It's obvious that in third case $W_{0}(s)$ is proper fraction and, as a result,

1. if $p \leq m+q-1$, then $\mu_{1}>\mu_{2}$ and $k_{d}=n-m$;

2. if $m+q-1 \leq p \leq 2 n-m+q-1$, then

$$
k_{d}=\left\{\begin{array}{l}
n+q-1-p, \text { if } m+q-1 \leq p<n+q-1, \\
0, \text { if } \mu_{1} \leq \mu_{2} .
\end{array}\right.
$$

3. if $p \geq 2 n-m+q-1$, then $\mu_{1}<\mu_{2}$ and $k_{d}=0$. These expressions imply necessary and sufficient condition of propriety of transfer function

$$
p-m-q+1>0 \text {. }
$$

If condition (15) is not fulfilled, transfer function (7) must be deformed by multiplying its numerator to $n^{*}=n+q-p-1$ additional terms

$$
H^{*}=H(s)\left(s+h_{1}^{2}\right)\left(s+h_{2}^{2}\right) \ldots\left(s+h_{n^{*}}^{2}\right),
$$

where parameters $h_{i}$ affect the effectiveness of the filter and can be also used for optimization.

Optimal solution of this problem can be treated as a tradeoff between maximization of response speed, minimization of overshoot and minimization of $\left|F\left(j \omega_{0}\right)\right|$. All these values are affected with parameters $k$ and $h_{i}$, used for computations (8)-(11), (16). As a result, original problem can be reformulated as the problem to minimize of the following value:

$$
\begin{aligned}
& J=J(W)=T_{p}-T_{p}^{0}+\left|T_{p}-T_{p}^{0}\right|+J_{p}-J_{p}^{0}+\ldots \\
& \ldots+\left|J_{p}+J_{p}^{0}\right|+\left|F\left(j \omega_{0}\right)\right|-F_{0}+|| F\left(j \omega_{0}\right)\left|-F_{0}\right|,
\end{aligned}
$$

where $T_{p}^{0}, J_{p}^{0}, F_{0}$ are desired values of transient period, overshoot and sensitivity to a disturbance respectively. $T_{p}=T_{p}(W), \quad J_{p}=J_{p}(W), \quad F\left(j \omega_{0}\right)$ are their values, obtained with filter $W(s)$. It is obvious, that minimal value of functional (17) is 0 , and $J(W)=0$ implies

$$
T_{p} \leq T_{p}^{0}, J_{p} \leq J_{p}^{0},\left|F\left(j \omega_{0}\right)\right| \leq F_{0} .
$$

Optimal parameters $k=k^{*}$ and $h_{i}=h_{i}^{*}$ can be computed with the following algorithm:

1. Construct the polynomials $A(s), \quad B(s), \quad P(s)$, $H(s)$. Check the condition (15) and, if necessary, calculate $n^{*}$.

2. Set initial coefficient of the intensity $k=k_{0}$ in the functional (5).

3. Set initial vector $h^{0}=\left(h_{1}^{0}, h_{2}^{0}, \ldots, h_{n^{*}}^{0}\right)$ and deform the spectral density (16).
4. Compute transfer function (11) of the corrector $\widetilde{W}(s)$.

5. Evaluate $J=J(\tilde{W})(17)$.

6. Minimize (17), repeating steps 3-5, with any numerical method of function minimization, e.g. NelderMead method, which is implied in the simulation example, described in Section 4. Obtain vector $\tilde{h}$ and filter $\widetilde{W}$.

7. If $J(\widetilde{W})$ is not close to zero, i.e. some of values exceed its desired values, then repeat 2-6 with new $k=\tilde{k}$. Optimal $k=k^{*}$ can be searched with any numerical method of function minimization or with enumeration.

8. Replicate step 4 with optimal parameters $k=k^{*}$, $h=h^{*}$, and obtain filter, satisfying conditions (18).

\section{Simulation results}

Let us consider the linear time invariant system (1), describing yaw motion of marine ship and the basic fault estimation observer with parameters [6]

$$
\begin{gathered}
\mathbf{A}=\left(\begin{array}{ccc}
-0.0936 & 0.634 & 0 \\
0.048 & -0.0717 & 0 \\
0 & 1 & 0
\end{array}\right), \mathbf{b}=\left(\begin{array}{c}
0.0196 \\
0.0160 \\
0
\end{array}\right), \\
\mathbf{E}=\mathbf{b}, \mathbf{g}=\left(\begin{array}{c}
-0.00016 \\
0.00000246 \\
0
\end{array}\right), \quad \mathbf{L}=\left(\begin{array}{c}
158.79 \\
117.05 \\
15.3
\end{array}\right), \quad \mathbf{l}_{\mathrm{f}}=31623 .
\end{gathered}
$$

Assume that the sea disturbance is $d(t)=400 \sin (0.65 t)$, i.e. disturbance is regular and its frequency $\omega_{0}=0.65$. Let assume the blurriness of the spectrum $s_{t}=0.01$, and the dispersion $D_{r}=1$ that implies

$$
H(s)=\frac{N_{d}(s)}{T_{d}(s)}=\frac{0.091}{s^{2}+0.013 s+0.423},
$$

and let consider execution of algorithm, described in previous Section.

1. Base polynomials from (6) and $n^{*}$ :

$$
\begin{gathered}
A(s)=s^{4}+0.811 s^{3}+0.037 s^{2}, \\
B(s)=-15.30 s^{3}-129.45 s^{2}-525.10 s-76.20, \\
P(s)=0.0000046 s^{2}-0.0000075 s, n^{*}=3 .
\end{gathered}
$$

2. Set initial parameters of deforming polynomial $h^{0}=\left(\begin{array}{lll}0.2 & 2 & 20\end{array}\right)$.

3. Optimal parameters $\quad k^{*}=5000$, $h^{0}=\left(\begin{array}{lll}1.57 & 27.65 & 0.2\end{array}\right)$.

4. Obtain a corrective filter with the transfer function

$$
W(s)=\frac{3.97 s^{5}+3.51 s^{4}+1.78 s^{3}+1.18 s^{2}+0.046 s}{s^{5}+709.7 s^{4}+4178^{3}+6367 s^{2}+3768 s+442.6} .
$$

Figure 1 allows to compare frequency responses of the base observer and the corrected one. It can be seen 
that the curve $|F(j \omega)|$ has a dip in the area of $\omega_{0}$, showing frequency properties of designed fault estimation observer.

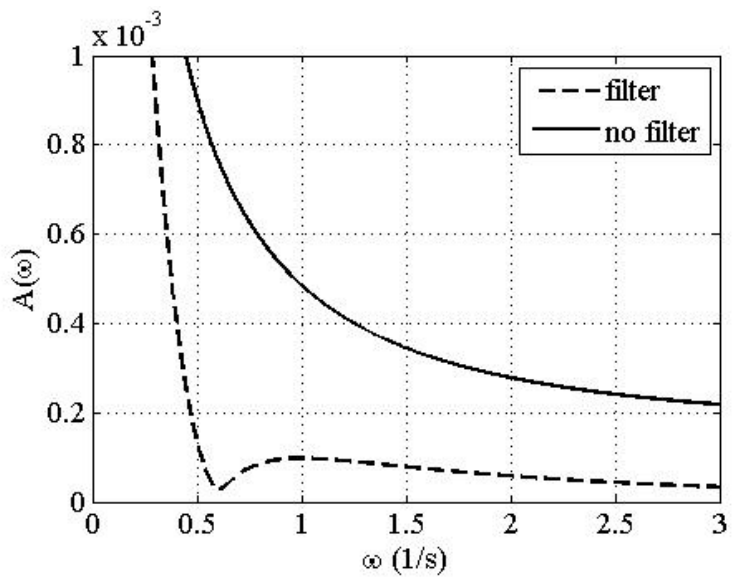

Figure 1. Comparison of frequency responses.

Figure 2 demonstrates the effectiveness of corrective filtering. It can be seen that initial observer generates signal with huge amplitude, when a fault is absent. When correction filter is switched on from the 100-th s, an error of fault estimation becomes close to zero. On the other hand, the fault at $200 \mathrm{~s}$ is successfully detected and estimated.

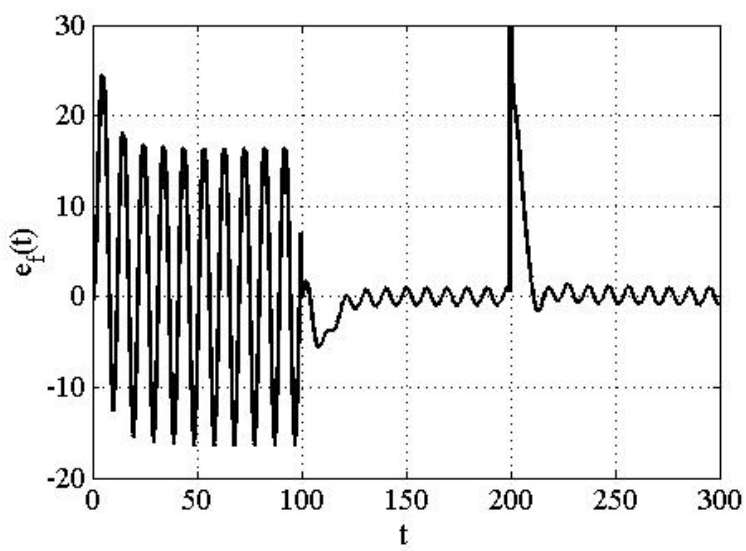

Figure 2. Signal $e_{f}(t)$ (error of fault estimation) generated by the basic adaptive observer $(0-100 \mathrm{~s})$ and corrected one with estimation of fault at $200 \mathrm{~s}$

\section{Conclusion}

This paper proposed a specific approach to the fault estimation in presence of external disturbances with the large amplitude. A design of the observer is realized in more extended area, than in most of the previous approaches, devoted to fault detection or estimation. It is significant that computational complexity of polynomial calculations is not very high, that may be crucial sometimes.

On the other hand, proposed approach has some disadvantages. It doesn't provide enough response speed and can't be applied to estimation of quickly varying faults. The object of future research is to overcome these problems.

\section{References}

1. K. Zhang, B. Jiang, P. Shi, Observer-based fault estimation and accomodation for dynamic systems (Springer 2012)

2. K. Zhang, B. Jiang, P. Shi, P., IET Control Theory \& Applications, 2, 189 (2009).

3. V. Serbak, P. Liscinsky, Advances in Electrical and Electronic Engineering, 1, 13 (2015)

4. A Drouot, H. Noura, L. Goerig, P. Piot, IFACPapersOnLine, 21, 180, (2015)

5. E. I. Veremey, Applied Mathematical Sciences, 79, 3897, (2015).

6. E.I. Veremey. Journal of Marine Science and Application, 13, 2, 127, (2014). 